\title{
Review Article \\ Partition Function in One, Two, and Three Spatial Dimensions from Effective Lagrangian Field Theory
}

\author{
Christoph P. Hofmann \\ Facultad de Ciencias, Universidad de Colima, Bernal Díaz del Castillo 340, 28045 Colima, COL, Mexico \\ Correspondence should be addressed to Christoph P. Hofmann; christoph.peter.hofmann@gmail.com
}

Received 9 December 2013; Accepted 3 February 2014; Published 11 March 2014

Academic Editors: Y. Miyano and M. Sanati

Copyright (C) 2014 Christoph P. Hofmann. This is an open access article distributed under the Creative Commons Attribution License, which permits unrestricted use, distribution, and reproduction in any medium, provided the original work is properly cited.

\begin{abstract}
The systematic effective Lagrangian method was first formulated in the context of the strong interaction; chiral perturbation theory (CHPT) is the effective theory of quantum chromodynamics (QCD). It was then pointed out that the method can be transferred to the nonrelativistic domain - in particular, to describe the low-energy properties of ferromagnets. Interestingly, whereas for Lorentzinvariant systems the effective Lagrangian method fails in one spatial dimension $\left(d_{s}=1\right)$, it perfectly works for nonrelativistic systems in $d_{s}=1$. In the present brief review, we give an outline of the method and then focus on the partition function for ferromagnetic spin chains, ferromagnetic films, and ferromagnetic crystals up to three loops in the perturbative expansion-an accuracy never achieved by conventional condensed matter methods. We then compare ferromagnets in $d_{s}=1,2,3$ with the behavior of QCD at low temperatures by considering the pressure and the order parameter. The two apparently very different systems (ferromagnets and QCD) are related from a universal point of view based on the spontaneously broken symmetry. In either case, the low-energy dynamics is described by an effective theory containing Goldstone bosons as basic degrees of freedom.
\end{abstract}

\section{Introduction}

While the methods used in particle physics tend to be rather different from the microscopic approaches taken by condensed matter physicists, there is though one fully systematic analytic method that can be applied to both sectors. The effective Lagrangian method, based on a symmetry analysis of the underlying theory, makes use of the fact that the low-energy dynamics is dominated by Goldstone bosons which emerge from the spontaneously broken symmetry: chiral symmetry $\mathrm{SU}(3)_{R} \times \mathrm{SU}(3)_{L} \rightarrow \mathrm{SU}(3)_{V}$ in quantum chromodynamics $(\mathrm{QCD})$ and spin rotation symmetry $\mathrm{O}(3) \rightarrow \mathrm{O}(2)$ in the context of ferromagnets. The method thus connects systems as disparate as QCD and ferromagnets from a universal point of view based on symmetry. The low-energy properties of the system are an immediate consequence of the spontaneously broken symmetry, while the specific microscopic details only manifest themselves in the values of a few effective constants. Still, as we are dealing with nonrelativistic kinematics in the case of the ferromagnet, apart from analogies, there are important differences: most remarkably, the effective
Lagrangian method, unlike for systems with relativistic kinematics, perfectly works for ferromagnets in one spatial dimension $\left(d_{s}=1\right)$.

While the low-temperature behavior of QCD was discussed more than two decades ago within effective field theory [1-3], the low-temperature properties of ferromagnetic crystals, films, and spin chains were considered only very recently within the effective Lagrangian framework [4-9]. In the present paper, we review both QCD and ferromagnets, trying to build a bridge between the particle physics and condensed matter communities. For example, so-called chiral logarithms, well known in Lorentz-invariant effective theories in $d=3+1$, also show up in the context of ferromagnets in $d=2+1$ dimensions (our notation is $d=d_{s}+1$, where $d_{s}$ is the spatial dimension).

In the first part of this review, an outline of the effective Lagrangian method is provided, covering both relativistic and nonrelativistic kinematics. In the second part we present the low-temperature expansions for the pressure and the order parameters, that is, the quark condensate in QCD and the spontaneous magnetization in the context of ferromagnets. 
In particular, by considering the suppression of loop diagrams and the absorption of ultraviolet divergences, we point out analogies and differences in the lowtemperature behavior of ferromagnetic systems and QCD.

As is well known, QCD-the theory of the strong interaction-cannot be solved perturbatively at low energies where the QCD coupling constant is not a small parameter. Therefore, its effective theory-chiral perturbation theory (CHPT) - based on an expansion in powers of energy and momenta, rather than on an expansion in powers of the QCD coupling constant, represents an indispensable tool to explore the low-energy domain of QCD. In the case of ferromagnets, the Heisenberg model can be solved at low energies by microscopic methods. Here, effective Lagrangians thus represent an alternative scheme which is, however, more efficient than conventional methods such as spin-wave theory.

A crucial point is that the effective Lagrangian technique is fully systematic and model independent and does not resort to any approximations or ad hoc assumptions. To appreciate the power of the effective method, we mention that-until Dyson's monumental work on the $d=3+$ 1 ferromagnet $[10,11]$-it was unclear at which order in the low-temperature expansion of the spontaneous magnetization the spin-wave interaction shows up. While the correct answer is $T^{4}[10-12]$, other researchers obtained $T^{7 / 4}$, $T^{2}$, and $T^{3}$ (see [13-24]). Within the effective Lagrangian framework, it was straightforward to confirm Dyson's result [4]. Likewise, the effective method allowed one to go beyond Dyson, demonstrating that the first interaction correction to $T^{4}$ is of order $T^{9 / 2}$ [5], whereas all other proposals in the literature, $T^{5}, T^{13 / 2}$, and $T^{15 / 2}$, are incorrect [25-27].

In $d_{s}=1,2$ the analogous question regarding the spin-wave interaction has largely been ignored in the many relevant articles [28-72], which are based on methods as diverse as spin-wave theory, Schwinger-boson mean-field theory, Green functions, scaling methods, and numerical simulations. The impact of the spin-wave interaction in ferromagnetic films and spin chains was first addressed systematically and conclusively solved with effective Lagrangians in [6-9].

\section{Effective Lagrangian Field Theory}

The link between the underlying, or microscopic, theoryQCD Lagrangian and Heisenberg Hamiltonian in the present context-and the effective theory is provided by symmetry. The effective action $\int d^{d} x \mathscr{L}_{\text {eff }}$ must be invariant under all the symmetries of the underlying theory [73]. The essential point is that the terms appearing in the effective Lagrangian $\mathscr{L}_{\text {eff }}$ can be organized according to the number of space and time derivatives that act on the Goldstone boson fields. At low energies, terms with few derivatives dominate the dynamics [73-75].
We first consider Lorentz-invariant theories. In quantum chromodynamics, the underlying Lagrangian is given by

$$
\mathscr{L}_{\mathrm{QCD}}=-\frac{1}{2 g^{2}} \operatorname{tr}_{c} G_{\mu \nu} G^{\mu \nu}+\bar{q} i \gamma^{\mu} D_{\mu} q-\bar{q} m q,
$$

where $g$ is the strong coupling constant, $q(x)$ is the quark field, $G_{\mu \nu}$ is the field strength of the gluon field, $m=$ $\operatorname{diag}\left(m_{u}, m_{d}, m_{s}, \ldots\right)$ is the quark mass matrix, and $\operatorname{tr}_{c}$ denotes the trace of a color matrix. In the chiral limit (i.e., when the quark masses $m_{u}, m_{d}, m_{s}$ are sent to zero) the above expression is invariant under the chiral transformation $\mathrm{SU}(3)_{R} \times \mathrm{SU}(3)_{L}$. The QCD vacuum, on the other hand, is only invariant under $\mathrm{SU}(3)_{V}$, such that the chiral symmetry is spontaneously broken. Goldstone's theorem $[76,77]$ then implies that we have eight pseudoscalar mesons in the lowenergy spectrum of QCD, which are identified with the three pions, the four kaons, and the $\eta$-particle. In the real world where the quark masses are different from zero, the chiral symmetry of the QCD Lagrangian is not exact, but only approximate. Therefore these particles are not strictly massless, but they represent the lightest degrees of freedom in the spectrum. Readers not familiar with QCD may consult the pedagogic [78] at this point. The derivative of the QCD Hamiltonian with respect to $m_{q}$ is the operator $\bar{q} q$. The corresponding derivative of the free energy density $z$ thus represents the expectation value of $\bar{q} q$, that is, the quark condensate,

$$
\langle\bar{q} q\rangle\left(T, m_{q}\right)=\frac{\partial z}{\partial m_{q}} .
$$

Spontaneous symmetry breaking is a prevalent phenomenon also in condensed matter physics, for example, in ferromagnets, which, on the microscopic level, are captured by the Heisenberg Hamiltonian augmented by the Zeeman term

$$
\mathscr{H}=-J \sum_{n . n .} \vec{S}_{m} \cdot \vec{S}_{n}-\mu \sum_{n} \vec{S}_{n} \cdot \vec{H}, \quad J=\text { const. }
$$

The magnetic field $\vec{H}$ couples with the vector of the total spin. The summation only extends over nearest neighbors, and the exchange coupling constant $J$ is purely isotropic. If the magnetic field is switched off, the Hamiltonian is symmetric under $O(3)$ spin rotations. The ground state of the ferromagnet $(J>0)$, however, is invariant under $O(2)$ only, such that the spin rotation symmetry is spontaneously broken.

The magnetic field $\vec{H}$ hence plays a role analogous to the quark masses $m_{q}$ : they are explicit symmetry breaking parameters. Likewise, the magnetization,

$$
\Sigma(T, H)=-\frac{\partial z}{\partial(\mu H)},
$$

is the analog of the quark condensate $\langle\bar{q} q\rangle\left(T, m_{q}\right)$. In particular, spontaneous magnetization (i.e., magnetization in the limit $\vec{H} \rightarrow 0$ ) corresponds to a nonzero value of the quark condensate in the chiral limit $m_{q} \rightarrow 0$. Both quantities are order parameters, their nonzero values signaling 
spontaneous symmetry breaking. Although the spontaneous symmetry breaking pattern $O(3) \rightarrow O(2)$ gives rise to two magnon fields according to Goldstone's theorem [76, 77], in a nonrelativistic setting only one type of spin-wave excitationor one magnon particle-exists in the low-energy spectrum of the ferromagnet [79-82]. Unlike in a Lorentz-invariant framework, there is no $1: 1$-correspondence between the number of Goldstone fields and Goldstone particles.

After this brief review of the underlying theories (QCD Lagrangian and Heisenberg Hamiltonian) we now proceed with the discussion of the corresponding effective theories. Chiral perturbation theory (CHPT) $[74,75]$ is well established in particle physics. It exploits the fact that the lowenergy dynamics of QCD is dominated by pions, kaons, and the $\eta$-particle, that is, the Goldstone bosons of the spontaneously broken chiral symmetry. These are the relevant degrees of freedom that appear in the effective Lagrangian $\mathscr{L}_{\text {eff. }}$. The terms in $\mathscr{L}_{\text {eff }}$ are organized according to the number of space-time derivatives: we are thus dealing with a derivative expansion, or equivalently, with an expansion in powers of momenta. The leading effective Lagrangian is of momentum order $p^{2}$

$$
\begin{aligned}
& \mathscr{L}_{\mathrm{eff}, \mathrm{QCD}}^{2}= \frac{1}{4} \mathscr{F}^{2} \operatorname{tr}\left(\partial_{\mu} U \partial^{\mu} U^{\dagger}\right) \\
&+\frac{1}{2} \mathscr{F}^{2} B \operatorname{tr}\left\{m\left(U+U^{\dagger}\right)\right\} \\
& U=\exp \left(\frac{i \pi^{a} \lambda_{a}}{\mathscr{F}}\right), \quad a=1, \ldots, 8,
\end{aligned}
$$

where the matrix $U$ contains the eight Goldstone boson fields $\pi^{a}$, with $\lambda_{a}$ as Gell-Mann matrices. The structure of the above terms is unambiguously fixed by chiral and Lorentz symmetry; these are the symmetries of the underlying theory which the effective theory inherits. Note that, at this order, there are two a priori unknown low-energy constants, $\mathscr{F}$ and $B$, that are not determined by the symmetries and hence have to be determined experimentally. Pseudoscalar mesons obey a relativistic dispersion law

$$
\begin{gathered}
\omega=\sqrt{c^{2} \vec{k}^{2}+c^{4} M_{\pi}^{2}}, \\
M_{\pi}^{2}=M^{2}+m_{1} M^{4}+m_{2} M^{6}+\mathcal{O}\left(M^{8}\right), \\
M^{2}=\left(m_{u}+m_{d}\right) B,
\end{gathered}
$$

where $M_{\pi}$ is the renormalized Goldstone boson mass and $m_{1}, m_{2}$ involve low-energy (or effective) constants from the subleading pieces $\mathscr{L}_{\text {eff, QCD }}^{4}$ and $\mathscr{L}_{\text {eff, QCD }}^{6}$ of the effective Lagrangian [3].

In [83], the effective Lagrangian method was transferred to nonrelativistic systems. A crucial point of that analysis is that for nonrelativistic kinematics, order parameters related to the generators of the spontaneously broken group show up as effective constants of a topological term which dominates the low-energy dynamics. This can not happen in a Lorentzinvariant setting [84]. For the ferromagnet, the leading contribution in $\mathscr{L}_{\text {eff, } F}$ is of momentum order $p^{2}$ [83]

$$
\mathscr{L}_{\mathrm{eff}, F}^{2}=\Sigma \frac{\epsilon_{a b} \partial_{0} U^{a} U^{b}}{1+U^{3}}+\Sigma \mu H U^{3}-\frac{1}{2} F^{2} \partial_{r} U^{i} \partial_{r} U^{i} .
$$

The effective constant of the topological term, involving one time derivative $\left(\partial_{0}\right)$ only, is the (zero-temperature) spontaneous magnetization $\Sigma$. The two real components of the magnon field, $U^{a}(a=1,2)$, are the first two components of the three-dimensional magnetization unit vector $U^{i}=$ $\left(U^{a}, U^{3}\right)$. Ferromagnetic magnons obey a quadratic dispersion law,

$$
\omega(\vec{k})=\gamma \vec{k}^{2}+\mu H+\gamma_{1} \vec{k}^{4}+\gamma_{2} \vec{k}^{6}+\mathcal{O}\left(\vec{k}^{8}\right), \quad \gamma=\frac{F^{2}}{\Sigma},
$$

where the coefficients $\gamma_{1}, \gamma_{2}$ contain higher-order effective constants from $\mathscr{L}_{\text {eff, } F}^{4}$ and $\mathscr{L}_{\text {eff, } F}^{6}$ [4]. It is important to note that, unlike in CHPT, time and space derivatives are not on the same footing in the case of nonrelativistic kinematics: two powers of momentum count as only one power of energy or temperature: $k^{2} \propto \omega, T$. Finally, we point out that the leading-order effective Lagrangian $\mathscr{L}_{\text {eff,F }}^{2}$ is space-rotation invariant, although the underlying Heisenberg model is not. Lattice anisotropies only start manifesting themselves in the next-to-leading piece $\mathscr{L}_{\text {eff, }}^{4}$ [85]. Still, here we assume that $\mathscr{L}_{\mathrm{eff}, F}^{4}$ and higher-order pieces in $\mathscr{L}_{\text {eff, } F}$ are space-rotation invariant-this idealization does not affect our conclusions.

\section{Ferromagnets and Quantum Chromodynamics}

While the low-temperature properties of QCD have been derived a long time ago within effective Lagrangian field theory [1-3], the analogous systematic three-loop analysis of ferromagnets in $d_{s}=1,2,3$ was performed only recently in [5, 7-9]. What is most remarkable from a conceptual point of view is that the effective method perfectly works in one spatial dimension in the case of nonrelativistic kinematics, whereas in a Lorentz-invariant setting the method fails in $d_{s}=1$. The reason is that the perturbative evaluation of the partition function is based on the suppression of loop diagrams by some power of momenta. This loop suppression depends both on the spatial dimension $d_{s}$ of the system and on the dispersion relation of its Goldstone bosons [8, 9]. For systems displaying a quadratic dispersion relation, a loop corresponds to the integral

$$
\int d \omega d^{d_{s}} k\left(\omega-\gamma \vec{k}^{2}\right)^{-1} \propto p^{d_{s}},
$$

which, on dimensional grounds, is proportional to $d_{s}$ powers of momentum. In particular, loops related to ferromagnetic spin chains are still suppressed by one power of momentum.

For systems with a linear (relativistic) dispersion relation, obeyed, for example, by the pseudoscalar mesons in the chiral 


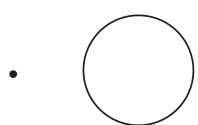

2

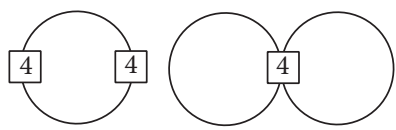

$9 \mathrm{~b}$

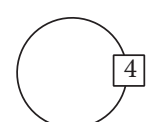

7

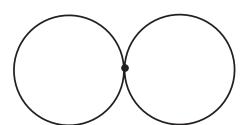

8
$9 \mathrm{a}$

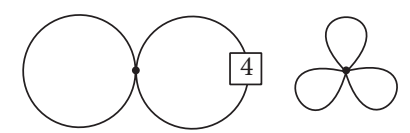

$10 \mathrm{~b}$

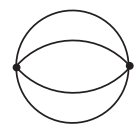

$11 \mathrm{c}$

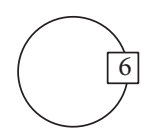

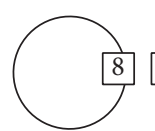

$11 \mathrm{~d}$

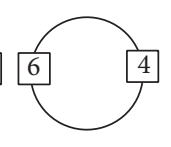

$11 \mathrm{e}$
FIGURE 1: Ferromagnet in three spatial dimensions: Feynman diagrams of the low-temperature expansion of the partition function up to three loops $\mathcal{O}\left(p^{11}\right)$. The vertices are numbered according to the piece of the effective Lagrangian $\mathscr{L}_{\text {eff }}$ they belong to. Vertices from the leading term $\mathscr{L}_{\text {eff }}^{2}$ are depicted by a filled circle. Loops in $d_{s}=3$ are suppressed by three powers of momentum.

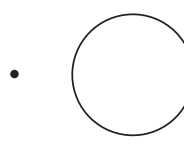

2

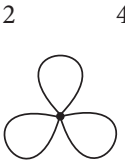

$8 \mathrm{a}$

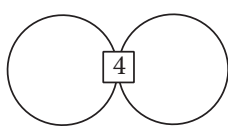

$8 d$

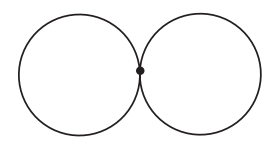

$6 a$

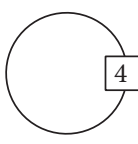

$6 \mathrm{~b}$

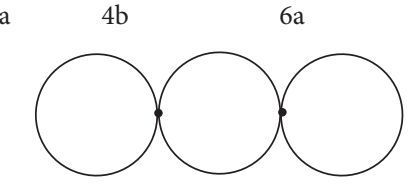

$8 \mathrm{~b}$

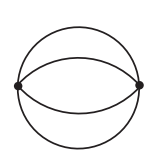

$8 \mathrm{c}$

FIGURE 2: Ferromagnet in two spatial dimensions and QCD: Feynman diagrams of the low-temperature expansion of the partition function up to three loops $\mathcal{O}\left(p^{8}\right)$. Loops in $d_{s}=2$ are suppressed by two powers of momentum. In the case of the ferromagnet, the temperature-independent diagrams $4 b, 6 c$, and 8 h do not occur [4].

limit, the loop suppression is rather different: there, a loop involves the integral

$$
\int d \omega d^{d_{s}} k\left(\omega^{2}-c^{2} \vec{k}^{2}\right)^{-1} \propto p^{d_{s}-1} .
$$

For Lorentz-invariant systems, loops in $d_{s}=3(2)$ are suppressed by two (one) powers of momentum. However, in $d_{s}=$ 1 , loops are not suppressed at all, and the effective method fails to systematically analyze Lorentz-invariant systems in one spatial dimension. These suppression rules are the basis for the organization of the Feynman graphs for the partition function of ferromagnets and QCD depicted in Figures 1-3.

The standard CHPT loop counting, depicted in Figure 2, corresponds to the loop counting for ferromagnets in two spatial dimensions. Here, each loop is suppressed by two powers of momentum. On general grounds, inspecting

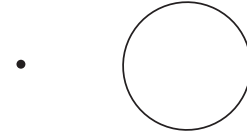

3

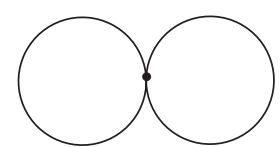

4

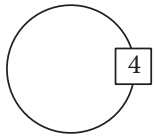

$5 d$

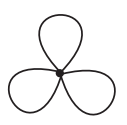

$5 \mathrm{a}$

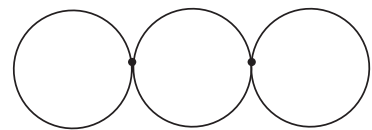

$5 \mathrm{~b}$

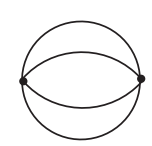

$5 c$
FIgURE 3: Ferromagnet in one spatial dimension: Feynman diagrams of the low-temperature expansion of the partition function up to three loops $\mathcal{O}\left(p^{5}\right)$. Loops in $d_{s}=1$ are suppressed by one momentum power.

Figures 1-3 reveals that in higher spatial dimensions, vertices involving subleading terms of the effective Lagrangian become more important. In $d_{s}=1, \mathscr{L}_{\text {eff }}^{4}$ only appears in a one-loop diagram (diagram $5 \mathrm{~d}$, Figure 3 ), such that the next-to-leading order effective constants in $\mathscr{L}_{\text {eff }}^{4}$ do not affect the spin-wave interaction. In $d_{s}=2,3$, however, $\mathscr{L}_{\text {eff }}^{4}$ also shows up in two-loop graphs and thus does contribute to the spin-wave interaction. While in $d_{s}=2$ these diagrams ( $8 \mathrm{~d}$ and $8 \mathrm{e}$ ) are of the same order as the three-loop graphs (diagrams 8a-c, Figure 2); in $d_{s}=3$ graphs 10a and 10b dominate over 11a-c. Furthermore, in $d_{s}=2$ we have insertions from $\mathscr{L}_{\text {eff }}^{6}$ and in $d_{s}=3$ even insertions from $\mathscr{L}_{\text {eff }}^{8}$. In higher spatial dimensions, the symmetries thus become less restrictive: more and more effective constants show up in a given thermodynamic quantity. Note however that the vertices which involve these higher-order effective constants from $\mathscr{L}_{\text {eff }}^{6}$ and $\mathscr{L}_{\text {eff }}^{8}$ only occur in one-loop diagrams and hence do not contribute to the spin-wave interaction up to the three-loop level we are considering here.

Before we present the results, we have to discuss an important issue, well known in chiral perturbation theory: graphs containing loops are divergent in the ultraviolet and one has to take care of these singularities through renormalization. The basic object which creates these singularities is the zero-temperature propagator at the origin: $\Delta(0)$. For Lorentz-invariant systems, the dimensionally regularized expression is

$$
\Delta(0)=(4 \pi)^{-d / 2} \Gamma\left(1-\frac{d}{2}\right) M^{d-2} .
$$

In a relativistic setting, UV-divergences hence arise in even space-time dimensions $d$. In CHPT, these singularities are absorbed by subleading effective constants in $\mathscr{L}_{\text {eff }}$, order by order in the derivative expansion in a systematic manner $[3,74,78]$. In the case of nonrelativistic kinematics, the situation is rather different. Regarding ferromagnets, the relevant expressions in the limit $\vec{x}, x_{4} \rightarrow 0$ (where $x_{4}$ is Euclidean time) vanish in dimensional regularization. As a consequence, only the temperature-dependent, that is, finite, pieces are relevant, such that here the handling of "UVdivergences" is much simpler [5, 7-9]. 
The cateye diagram-graph (11c, 8c, 5c) of Figures 1, 2, and 3-however is more involved; as it does not factorize into products of $\Delta(0)$ or derivatives thereof, the structure of the corresponding UV-divergences is more complicated. In chiral perturbation theory, the ultraviolet divergences of graph $8 \mathrm{c}$ are absorbed by next-to-leading order (NLO) effective constants from $\mathscr{L}_{\text {eff }}^{4}$ contained in the two-loop diagrams $8 \mathrm{~d}$ and $8 \mathrm{e}$. These NLO constants then undergo logarithmic renormalization [3]. For the ferromagnet in $d_{s}$ spatial dimensions, the cateye diagram is proportional to

$$
T^{d_{s}+2}(\mu H)^{\left(d_{s}-2\right) / 2}\left\{\sum_{n=1}^{\infty} \frac{e^{-\mu H n / T}}{n^{\left(d_{s}+2\right) / 2}}\right\}^{2} \Gamma\left(1-\frac{d_{s}}{2}\right) .
$$

In the case of ferromagnetic films $\left(d_{s} \rightarrow 2\right)$, the above regularized expression is divergent. Much like in chiral perturbation theory, the UV-singularity is absorbed by $\mathscr{L}_{\text {eff }}^{4}$-constants which are renormalized logarithmically. On the other hand, in one and three spatial dimensions, the $\Gamma$-function does not develop a pole, and the next-to-leading order effective constants do not undergo logarithmic renormalization-they are finite as they stand.

The fact that there are no ultraviolet singularities in $d_{s}=$ 1,3 is crucial for the nonrelativistic effective framework to be consistent; in $d_{s}=1$ (Figure 3 ) and $d_{s}=3$ (Figure 1 ), the cateye graph is of different order than the two-loop contributions with vertices from $\mathscr{L}_{\text {eff }}^{4}$. In contrast to $d_{s}=$ 2 , where three-loop and two-loop graphs "communicate" (they are of the same momentum order $p^{8}$ ) and the UVsingularities can thus be absorbed by $\mathscr{L}_{\text {eff }}^{4}$ constants, the effective loop analysis appears to be inconsistent in $d_{s}=$ 1,3: there are no two-loop diagrams available to absorb the "divergences" of the cateye graphs 11c (Figure 1) and $5 c$ (Figure 3 ). The puzzle is solved by noticing that in $d_{s}=1,3$, the cateye graph is not divergent and that the perturbative scheme hence is perfectly consistent.

We end our discussion of partition function diagrams by pointing out that, on the two-loop level, there is an important difference between chiral perturbation theory and ferromagnets. While graph 6a (Figure 2) in CHPT does contribute to the partition function, the same diagram for ferromagnets in any $d_{s}$ (i.e., including graph 8 of Figure 1 and graph 4 of Figure 3) turns out to be zero because of parity $[4,6,8,9]$. As a consequence, in the thermodynamic quantities the spin-wave interaction, in general, is weaker than the interaction among the pseudoscalar mesons.

The fact that the two-loop graph in question does not contribute in the case of the ferromagnet may be rather surprising to readers trained in chiral perturbation theory, where this two-loop graph indeed does contribute to the partition function [3]. We are dealing here with one of the novel effects that occur in the case of nonrelativistic kinematics. In fact, any of the three terms in the leading-order effective Lagrangian (7) yields expressions with four magnon fields that appear to lead to a nonzero contribution for the two-loop graph. However, making use of the leading-order equation for the magnon propagator-much like using the leadingorder equation for the pion propagator in chiral perturbation theory-these different contributions can be reduced to a single one, proportional to $\left[\partial_{r} G(x)\right]_{x=0}\left[\partial_{r} G(x)\right]_{x=0}$, where the time derivative and the magnetic field have been eliminated. One therefore concludes that this two-loop graph, in the context of ferromagnets, does not contribute to the partition function due to parity. On the other hand, the same twoloop diagram in chiral perturbation theory is proportional to $M^{2}[G(x)]_{x=0}[G(x)]_{x=0}$.

We now consider the low-temperature series for the pressure, starting with the ferromagnet. While the leading terms in the pressure $P_{d_{s}}$ are of order $\left(T^{5 / 2}, T^{2}, T^{3 / 2}\right)$ in $d_{s}=(3,2,1)$, the spin-wave interaction only emerges at $\left(T^{5}, T^{4} \ln T, T^{5 / 2}\right)$

$$
\begin{gathered}
P_{3}=a_{0} T^{5 / 2}+a_{1} T^{7 / 2}+a_{2} T^{9 / 2}+\mathbf{a}_{3} \mathbf{T}^{5} \\
+\mathbf{a}_{\mathbf{4}} \mathbf{T}^{\mathbf{1 1 / 2}}+\mathcal{O}\left(\mathbf{p}^{12}\right), \\
P_{2}=\widehat{a}_{0} T^{2}+\widehat{a}_{1} T^{3}+\widehat{\mathbf{a}}_{2}^{\mathbf{A}} \mathbf{T}^{4}+\widehat{\mathbf{a}}_{2}^{\mathbf{B}} \mathbf{T}^{4} \ln \mathbf{T}+\mathcal{O}\left(\mathbf{p}^{10}\right), \\
P_{1}=\widetilde{a}_{0} T^{3 / 2}+\widetilde{\mathbf{a}}_{1} \mathbf{T}^{5 / 2}+\mathcal{O}\left(\mathbf{p}^{6}\right) .
\end{gathered}
$$

Note that all interaction terms are boldfaced. Since the two-loop diagram with an insertion from $\mathscr{L}_{\text {eff, } F}^{2}$ does not contribute (diagram 6a in Figure 2), interaction terms of order $\left(T^{4}, T^{3}, T^{2}\right)$ in $d_{s}=(3,2,1)$ are absent. One further notices that the higher the spatial dimension is, the less important the interaction becomes; there are more terms related to noninteracting magnons, until the interaction sets in. In particular, as Dyson showed a long time ago [10, 11], the spin-wave interaction in the case of the three-dimensional ferromagnet only manifests itself at order $T^{5}$ in the pressure, that is, far beyond the leading Bloch term of order $T^{5 / 2}$.

Interestingly, ferromagnets in two spatial dimensions develop a logarithm, because in the limit $\vec{H} \rightarrow 0$ the cateye graph diverges logarithmically like $T^{4} \ln (\mu H / T)$ [7]. In chiral perturbation theory, the situation is analogous; in the chiral limit $\left(m_{q}, M_{\pi} \rightarrow 0\right)$, the cateye graph diverges like $T^{8} \ln \left(M_{\pi} / T\right)$ [3], such that we also have a logarithmic contribution in the low-temperature series of the pressure

$$
P_{\mathrm{QCD}}=b_{0} T^{4}+\mathbf{b}_{\mathbf{1}} \mathbf{T}^{\mathbf{6}}+\mathbf{b}_{\mathbf{2}} \mathbf{T}^{\mathbf{8}} \ln \mathbf{T}+\mathcal{O}\left(\mathbf{p}^{\mathbf{1 0}}\right) .
$$

But, unlike for $d_{s}=2$ ferromagnets, an analogous term $T^{8}$ (without $\ln$ ) is absent. This is because in chiral perturbation theory all effective $\mathscr{L}_{\text {eff }}^{4}$ constants undergo logarithmic renormalization, whereas for $d_{s}=2$, ferromagnets, only part of them [7].

In $d_{s}=1,3$, the leading terms involve half-integer powers of the temperature, while loops are further suppressed by powers of $p \propto T^{1 / 2}\left(p^{3} \propto T^{3 / 2}\right)$ in $d_{s}=1(3)$. For odd spatial dimensions, the pressure for ferromagnets thus exhibits both integer and half-integer powers of $T$. In the case of ferromagnetic films, on the other hand, the leading term in the pressure is of order $T^{2}$. Since here loops are suppressed by one power of $T$, the series does not involve any half-integer temperature powers. However, as we have pointed out before, logarithmic contributions emerge. In the limit $H, m_{q} \rightarrow 0$, 
the coefficients $a_{i}, \widehat{a}_{i}, \tilde{a}_{i}, b_{i}$ formally become $T$-independent, while for $H, m_{q} \neq 0$, they are complicated functions of the ratios $\mu H / T$ and $M_{\pi} / T$ [3, 5, 7-9]. Note however, as we discuss below, that the limit $H \rightarrow 0$ is problematic in $d_{s} \leq 2$.

Finally, the low-temperature series of the magnetization of ferromagnets,

$$
\bar{\Sigma}_{d_{s}}=\frac{\Sigma(T, H)}{\Sigma}
$$

in three, two, and one spatial dimensions, amount to

$$
\begin{gathered}
\bar{\Sigma}_{3}=1-\alpha_{0} T^{3 / 2}-\alpha_{1} T^{5 / 2}-\alpha_{2} T^{7 / 2} \\
-\boldsymbol{\alpha}_{3} \mathbf{T}^{\mathbf{4}}-\boldsymbol{\alpha}_{\mathbf{4}} \mathbf{T}^{\mathbf{9} / 2}+\mathcal{O}\left(\mathbf{T}^{5}\right), \\
\bar{\Sigma}_{2}=1-\widehat{\alpha}_{0} T-\widehat{\alpha}_{1} T^{2}-\widehat{\boldsymbol{\alpha}}_{2}^{\mathbf{A}} \mathbf{T}^{3}-\widehat{\boldsymbol{\alpha}}_{2}^{\mathbf{B}} \mathbf{T}^{3} \ln \mathbf{T}+\mathcal{O}\left(\mathbf{T}^{\mathbf{4}}\right), \\
\bar{\Sigma}_{1}=1-\widetilde{\alpha}_{0} T^{1 / 2}-\widetilde{\boldsymbol{\alpha}}_{\mathbf{1}} \mathbf{T}^{3 / 2}+\mathcal{O}\left(\mathbf{T}^{2}\right) .
\end{gathered}
$$

The low-temperature expansion for the analogous quantity in QCD, the quark condensate

$$
\frac{\langle\bar{q} q\rangle\left(T, m_{q}\right)}{\langle 0|\bar{q} q| 0\rangle}
$$

reads

$$
1-\beta_{0} T^{2}-\beta_{1} \mathbf{T}^{4}-\beta_{2} \mathbf{T}^{6} \ln \mathbf{T}+\mathcal{O}\left(\mathbf{T}^{8}\right)
$$

Again, for nonzero magnetic field and nonzero quark masses, the coefficients $\alpha_{i}, \widehat{\alpha}_{i}, \widetilde{\alpha}_{i}$, and $\beta_{i}$ depend on the ratios $\mu H / T$ and $M_{\pi} / T$ in a nontrivial way. In the chiral limit $\left(m_{q}, M_{\pi} \rightarrow\right.$ 0 ), the leading coefficient $\beta_{0}$ in the quark condensate reduces to $1 / 8 \mathscr{F}^{2}$. The leading coefficient in the spontaneous magnetization of the $d_{s}=3$ ferromagnet becomes $\alpha_{0}=$ $\zeta(3 / 2) / 8 \pi^{3 / 2} \Sigma \gamma^{3 / 2}$. The temperature scale in chiral perturbation theory is thus given by $\Lambda_{\mathrm{QCD}}^{T}=\sqrt{8} \mathscr{F} \approx 250 \mathrm{MeV}$, which roughly corresponds to the temperature where the chiral phase transition takes place [3]. In the case of the $d_{s}=3$ ferromagnet, the temperature scale is $\Lambda_{F}^{T}=$ $\alpha_{0}^{-2 / 3} \approx 10 \mathrm{meV}$, which is roughly of the order of the Curie temperature where the spontaneous magnetization becomes zero. Although these scales differ in more than ten orders of magnitude, the effective theory captures both systems from a universal perspective based on symmetry. In particular, for temperatures small compared to the corresponding scales $\Lambda_{\mathrm{QCD}}^{T}$ and $\Lambda_{F}^{T}$, the series presented in this review are perfectly valid.

It is important to point out that in one and two spatial dimensions, the leading coefficients $\widehat{\alpha}_{0}$ and $\widetilde{\alpha}_{0}$ in the magnetization become divergent in the limit $\vec{H} \rightarrow 0$. This is related to the Mermin-Wagner theorem [86], stating that spontaneous symmetry breaking in $d_{s} \leq 2$ cannot occur at finite temperature in the Heisenberg model. According to (16), the magnetization $\Sigma(T, H)$, in any dimension $d_{s}=$ $1,2,3$, tends to zero at a "critical" temperature $T_{c}$, where the spontaneously broken symmetry is restored. While $T_{c}$ in $d_{s}=$ 3 tends to a finite value in the limit $\vec{H} \rightarrow 0$ (much like for the quark condensate, $T_{c}$ tends to a finite value in the chiral limit), the "critical" temperatures in $d_{s}=1,2$ tend to zero if the magnetic field is switched off. Spontaneous symmetry breaking never occurs here at finite temperature-that is how the effective theory "knows" about the Mermin-Wagner theorem.

In addition, in $d_{s} \leq 2$, an energy gap is generated nonperturbatively at finite temperature [47, 87]. Strictly speaking this also implies that the limit $\vec{H} \rightarrow 0$ cannot be taken in $d_{s} \leq 2$, neither in the pressure nor in the magnetization, because we would then leave the domain of validity where the effective expansion applies [6-9]. Still, in two spatial dimensions, where the the nonperturbatively generated correlation length $\xi_{n p}$ is exponentially large $\left(\xi_{\mathrm{np}} \propto e^{1 / T}\right)$, this effect is tiny in the pressure and does not numerically affect the corresponding low-temperature series. In the context of ferromagnetic spin chains, however, where the nonperturbatively generated correlation length is proportional to the inverse temperature $\left(\xi_{\mathrm{np}} \propto 1 / T\right)$, it would be completely inconsistent to switch off the magnetic field even in the low-temperature series of the pressure [6-9].

It is important to point out that these subtleties in one and two spatial dimensions only emerge at finite temperature. In particular, at $T=0$ the limit $\vec{H} \rightarrow 0$ is well defined in $d_{s} \leq 2$. Much like in chiral perturbation theory or in ferromagnetic systems in $d_{s}=3$, no divergent behavior of the observables occurs. In other words, the "chiral limit" $\vec{H} \rightarrow 0$ is not problematic for ferromagnets living in one or two spatial dimensions at zero temperature. However, if the temperature is finite, then the magnetic field cannot be totally switched off in $d_{s} \leq 2$, because one would then leave the domain of validity of the effective expansion. As discussed in detail in [6-9], this parameter regime, where the effective expansion breaks down, is tiny. Again, this observation is related to the MerminWagner theorem and the nonperturbatively generated energy gap.

\section{Conclusions}

The systematic effective Lagrangian method is a very useful tool also in the nonrelativistic domain where it even works in one spatial dimension. The method is appealing due to its universal character, interconnecting different branches of physics, such as particle and condensed matter physics. Chiral logarithms showing up in the renormalization of next-toleading order effective constants in chiral perturbation theory and $d_{s}=2$ ferromagnets, for example, are due to analogous ultraviolet divergences. In general, the structure of the lowtemperature series-both in relativistic and nonrelativistic effective field theory-is an immediate consequence of the spontaneously broken symmetry. Regarding ferromagnets, the effective method proves more powerful than conventional condensed matter approaches, where analogous three-loop calculations are either missing or are erroneous. 


\section{Conflict of Interests}

The author, Christoph P. Hofmann, declares that there is no conflict of interests regarding the publication of this paper.

\section{References}

[1] J. Gasser and H. Leutwyler, "Light quarks atlow temperatures," Physics Letters B, vol. 184, no. 1, pp. 83-88, 1987.

[2] J. Gasser and H. Leutwyler, "Thermodynamics of chiral symmetry," Physics Letters B, vol. 188, no. 4, pp. 477-481, 1987.

[3] P. Gerber and H. Leutwyler, "Hadrons below the chiral phase transition," Nuclear Physics B, vol. 321, no. 2, pp. 387-429, 1989.

[4] C. P. Hofmann, "Spontaneous magnetization of the $\mathrm{O}(3)$ ferromagnet at low temperatures," Physical Review B, vol. 65, no. 9, Article ID 094430, 12 pages, 2002.

[5] C. P. Hofmann, "Spontaneous magnetization of an ideal ferromagnet: beyond Dyson's analysis," Physical Review B, vol. 84, no. 6, Article ID 064414, 16 pages, 2011.

[6] C. P. Hofmann, "Low-temperature properties of two- dimensional ideal ferromagnets," Physical Review B, vol no. 5, Article ID 054409, 10 pages, 2012.

[7] C. P. Hofmann, "Thermodynamics of two-dimensional ideal ferromagnets: three-loop analysis," Physical Review B, vol. 86, no. 18, Article ID 184409, 14 pages, 2012.

[8] C. P. Hofmann, "Low-temperature properties of ferromagnetic spin chains in a magnetic field," Physical Review B, vol. 87, no. 18, Article ID 184420, 12 pages, 2013.

[9] C. P. Hofmann, "Thermodynamics of ferromagnetic spin chains in a magnetic field: impact of the spin-wave interaction," Physica B: Condensed Matter, 2014.

[10] F. J. Dyson, "General theory of spin-wave interactions," Physical Review, vol. 102, no. 5, pp. 1217-1230, 1956.

[11] F. J. Dyson, "Thermodynamic behavior of an ideal ferromagnet," Physical Review, vol. 102, no. 5, pp. 1230-1244, 1956.

[12] J. Zittartz, "On the spin wave problem in the Heisenberg model of Ferromagnetism," Zeitschrift für Physik, vol. 184, no. 5, pp. 506-520, 1965.

[13] H. A. Kramers, Communications from the Kamerlingh Onnes Laboratory of the University of Leiden, vol. 22, Kamerlingh Onnes Laboratorium, 1936.

[14] W. Opechowski, "Über die temperaturabhängigkeit der magnetisierung eines ferromagnetikums bei tiefen temperaturen," Physica, vol. 4, no. 8, pp. 715-722, 1937.

[15] M. R. Schafroth, "Self-consistent spin-wave theory for the ferromagnetic exchange problem," Proceedings of the Royal Society A, vol. 67, no. 1, p. 33, 1954.

[16] J. van Kranendonk, "Theory of the low-temperature properties of ferromagnetic crystals," Physica, vol. 21, pp. 81-82, 1955.

[17] J. van Kranendonk, "Spin-deviation theory of ferromagnetism I general theory," Physica, vol. 21, pp. 749-766, 1955.

[18] J. van Kranendonk, "Spin-deviation theory of ferromagnetism. II. The non-ideal spin-deviation gas," Physica, vol. 21, pp. 925945, 1955

[19] I. Mannari, "Quantization of spin wave field," Progress of Theoretical Physics, vol. 19, no. 2, pp. 201-213, 1958.

[20] R. Brout and H. Haken, "Theory of the magnetization curve of a Heisenberg ferromagnet," Bulletin of the American Physical Society, vol. 5, p. 148, 1960.
[21] R. A. Tahir-Kheli and D. ter Haar, "Use of green functions in the theory of ferromagnetism. I. General discussion of the spin-S case," Physical Review, vol. 127, no. 1, pp. 88-94, 1962.

[22] R. B. Stinchcombe, G. Horwitz, F. Englert, and R. Brout, "Thermodynamic behavior of the heisenberg ferromagnet," Physical Review, vol. 130, no. 1, pp. 155-176, 1963.

[23] H. B. Callen, "Green function theory of ferromagnetism," Physical Review, vol. 130, no. 3, pp. 890-898, 1963.

[24] T. Oguchi and A. Honma, "Theory of ferro- and antiferromagnetism by the method of green functions," Journal of Applied Physics, vol. 34, no. 4, pp. 1153-1160, 1963.

[25] T. Morita and T. Tanaka, "Effect of $n$-spin-wave interaction on the low-temperature spontaneous magnetization," Journal of Mathematical Physics, vol. 6, no. 7, pp. 1152-1154, 1965.

[26] C.-C. Chang, "Free energy of the three-dimensional spin-1/2 quantum Heisenberg model to $\mathrm{O}\left[\mathrm{T}^{6}\right]$," Annals of Physics, vol. 293, no. 2, pp. 111-125, 2001.

[27] J. Achleitner, "Magnetization and magnon excitation energies of the magnetic semiconductor EuTe," Modern Physics Letters B, vol. 25, no. 24, pp. 1925-1938, 2011.

[28] V. Mubayi and R. V. Lange, "Phase transition in the twodimensional heisenberg ferromagnet," Physical Review, vol. 178, no. 2, pp. 882-894, 1969.

[29] J. H. P. Colpa, "On the heat capacity of the (nearly) quadratic heisenberg, $\mathrm{S}=12$, ferromagnet," Physica, vol. 57, no. 3, pp. 347380, 1972.

[30] M. Takahashi, "One-dimensional Heisenberg model at finite temperature," Progress of Theoretical Physics, vol. 46, no. 2, pp. 401-415, 1971.

[31] J. Kondo and K. Yamaji, "Green's-function formalism of the onedimensional Heisenberg spin system," Progress of Theoretical Physics, vol. 47, no. 3, pp. 807-818, 1972.

[32] M. Takahashi, "Low-temperature specific heat of spin-1/2 anisotropic Heisenberg ring," Progress of Theoretical Physics, vol. 50, no. 5, pp. 1519-1536, 1973.

[33] K. Yamaji and J. Kondo, "Thermodynamics of the twodimensional ferromagnetic Heisenberg spin systems," Physics Letters A, vol. 45, no. 4, pp. 317-318, 1973.

[34] J. J. Cullen and D. P. Landau, "Monte Carlo studies of onedimensional quantum Heisenberg and XY models," Physical Review B, vol. 27, no. 1, pp. 297-313, 1983.

[35] J. W. Lyklema, "Monte Carlo study of the one-dimensional quantum Heisenberg ferromagnet near $\mathrm{T}=0$," Physical Review $B$, vol. 27, no. 5, pp. 3108-3110, 1983.

[36] P. Schlottmann, "Critical behavior of the isotropic ferromagnetic quantum Heisenberg chain," Physical Review Letters, vol. 54, no. 19, pp. 2131-2134, 1985.

[37] M. Takahashi and M. Yamada, "Spin-1/2 one-dimensional Heisenberg ferromagnet at low-temperature," Journal of the Physical Society of Japan, vol. 54, no. 8, pp. 2808-2811, 1985.

[38] M. Yamada and M. Takahashi, "Critical behavior of spin-1/2 one-dimensional Heisenberg ferromagnet at low temperatures," Journal of the Physical Society of Japan, vol. 55, no. 6, pp. 20242036, 1986.

[39] M. Takahashi, "Quantum Heisenberg ferromagnets in one and two dimensions at low temperature," Progress of Theoretical Physics Supplement, vol. 87, pp. 233-246, 1986.

[40] S. Kadowaki and A. Ueda, "A direct evaluation method of the partition function of quantum spin systems," Progress of Theoretical Physics, vol. 75, no. 2, pp. 451-454, 1986. 
[41] P. Schlottmann, "Low-temperature behavior of the $S=(1 / 2)$ ferromagnetic Heisenberg chain," Physical Review B, vol. 33, no. 7, pp. 4880-4886, 1986.

[42] K.-J. Lee and P. Schlottmann, "Critical behavior of the isotropic ferromagnetic Heisenberg chain with arbitrary spin S," Physical Review B, vol. 36, no. 1, pp. 466-473, 1987.

[43] M. Takahashi, "Few-dimensional Heisenberg ferromagnets at low temperature," Physical Review Letters, vol. 58, no. 2, pp. 168170, 1987.

[44] M. Takahashi, "Two-dimensional Heisenberg ferromagnet," Japanese Journal of Applied Physics, vol. 26, supplement 26-3-1, pp. 869-870, 1987.

[45] Y. C. Chen, H. H. Chen, and F. Lee, "Quantum monte carlo study of the one-dimensional exchange interaction model," Physics Letters A, vol. 130, no. 4-5, pp. 257-259, 1988.

[46] L. S. Campana, A. Caramico D'Auria, U. Esposito, and G. Kamieniarz, "Thermodynamic functions of the $S=1 / 2$ onedimensional ferromagnet via the renormalization-group approach and Greens function technique," Physical Review B, vol. 39, no. 13, pp. 9224-9230, 1989.

[47] P. Kopietz, "Low-temperature behavior of the correlation length and the susceptibility of the ferromagnetic quantum Heisenberg chain," Physical Review B, vol. 40, no. 7, pp. 5194-5197, 1989.

[48] M. Takahashi, "Comment on finite 2D quantum Heisenberg ferromagnet," Progress of Theoretical Physics, vol. 83, no. 5, pp. 815-818, 1990.

[49] A. Auerbach and D. P. Arovas, "New approaches to the quantum Heisenberg models: Schwinger boson representations (invited)," Journal of Applied Physics, vol. 67, no. 9, pp. 57345739, 1990.

[50] A. Auerbach and D. P. Arovas, "Schwinger Boson mean field theory of the quantum Heisenberg model," in Field Theories in Condensed Matter Physics, Z. Tesanovich, Ed., p. 1, AddisonWesley, Reading, Mass, USA, 1990.

[51] M. Yamada, "Thermal Bethe Ansatz study of correlation length of spin-1/2 Heisenberg ferromagnetic chain," Journal of the Physical Society of Japan, vol. 59, no. 3, pp. 848-856, 1990.

[52] T. Delica and H. Leschke, "Formulation and numerical results of the transfer-matrix method for quantum spin chains," Physica A, vol. 168, no. 2, pp. 736-767, 1990.

[53] D. A. Yablonskiy, "Tyablikov approximation in the theory of low-dimensional quantum Heisenberg ferromagnets and antiferromagnets," Physical Review B, vol. 44, no. 9, pp. 44674472, 1991.

[54] F. Suzuki, N. Shibata, and C. Ishii, "Thermodynamics of lowdimensional Heisenberg ferromagnets by the Green's function method," Journal of the Physical Society of Japan, vol. 63, no. 4, pp. 1539-1547, 1994.

[55] H. Nakano and M. Takahashi, "Quantum Heisenberg model with long-range ferromagnetic interactions," Physical Review B, vol. 50, no. 14, pp. 10331-10334, 1994.

[56] H. Nakamura and M. Takahashi, "The free energy and the scaling function of the ferromagnetic Heisenberg chain in a magnetic field," Journal of the Physical Society of Japan, vol. 63, no. 7, pp. 2563-2571, 1994.

[57] H. Nakamura, N. Hatano, and M. Takahashi, "Universal finitesize scaling function of the ferromagnetic Heisenberg chain in a magnetic field," Journal of the Physical Society of Japan, vol. 64, no. 6, pp. 1955-1966, 1995.

[58] H. Nakamura, N. Hatano, and M. Takahashi, "Universal finitesize scaling function of the ferromagnetic Heisenberg chain in a magnetic field. II-nonlinear susceptibility," Journal of the Physical Society of Japan, vol. 64, no. 11, pp. 4142-4155, 1995.

[59] N. Read and S. Sachdev, "Continuum quantum ferromagnets at finite temperature and the quantum hall effect," Physical Review Letters, vol. 75, no. 19, pp. 3509-3512, 1995.

[60] M. Takahashi, H. Nakamura, and S. Sachdev, "Universal lowtemperature properties of quantum and classical ferromagnetic chains," Physical Review B, vol. 54, no. 2, pp. R744-R747, 1996.

[61] A. W. Sandvik, R. R. P. Singh, and D. K. Campbell, "Quantum Monte Carlo in the interaction representation: application to a spin-Peierls model," Physical Review B, vol. 56, no. 22, pp. 1451014528, 1997.

[62] M. Hamedoun, Y. Cherriet, A. Hourmatallah, and N. Benzakour, "Quantum Heisenberg model with long-range ferromagnetic interactions: a Green's function approach," Physical Review B, vol. 63, no. 17, Article ID 172402, 2001.

[63] M. Kollar, I. Spremo, and P. Kopietz, "Spin-wave theory at constant order parameter," Physical Review B, vol. 67, no. 10, Article ID 104427, 2003.

[64] I. Junger, D. Ihle, J. Richter, and A. Klümper, "Green-function theory of the Heisenberg ferromagnet in a magnetic field," Physical Review B, vol. 70, no. 10, Article ID 104419, 2004.

[65] S.-J. Gu, N. M. R. Peres, and Y.-Q. Li, "Numerical and Monte Carlo Bethe ansatz method: 1D Heisenberg model," European Physical Journal B, vol. 48, no. 2, pp. 157-165, 2005.

[66] D. V. Dmitriev and V. Y. Krivnov, "Frustrated ferromagnetic spin-1/2 chain in a magnetic field," Physical Review B, vol. 73, no. 2, Article ID 024402, 2006.

[67] J. Sirker and M. Bortz, "Quantum versus classical behavior in the boundary susceptibility of the ferromagnetic Heisenberg chain," Physical Review B, vol. 73, no. 1, Article ID 014424, 2006.

[68] X. W. Guan, M. T. Batchelor, and M. Takahashi, "Ferromagnetic behavior in the strongly interacting two-component Bose gas," Physical Review A, vol. 76, Article ID 043617, 11 pages, 2007.

[69] T. N. Antsygina, M. I. Poltavskaya, I. I. Poltavsky, and K. A. Chishko, "Thermodynamics of low-dimensional spin- $1 / 2$ Heisenberg ferromagnets in an external magnetic field within a Green function formalism," Physical Review B, vol. 77, no. 2, Article ID 024407, 2008.

[70] I. Juhász Junger, D. Ihle, L. Bogacz, and W. Janke, “Thermodynamics of Heisenberg ferromagnets with arbitrary spin in a magnetic field," Physical Review B, vol. 77, no. 17, Article ID 174411, 15 pages, 2008.

[71] M. W. Liu, Y. Chen, C. C. Song, Y. Wu, and H. L. Ding, "The magnetic properties of one-dimensional spin-1 ferromagnetic Heisenberg model in a magnetic field within Callen approximation," Solid State Communications, vol. 151, no. 6, pp. 503-508, 2011.

[72] D. V. Dmitriev and V. Ya. Krivnov, "Universal low-temperature magnetic properties of the classical and quantum dimerized ferromagnetic spin chain," Physical Review B, vol. 86, no. 13, Article ID 134407, 9 pages, 2012.

[73] S. Weinberg, "Phenomenological Lagrangians," Physica A, vol. 96, no. 1-2, pp. 327-340, 1979.

[74] J. Gasser and H. Leutwyler, "Chiral perturbation theory to one loop," Annals of Physics, vol. 158, no. 1, pp. 142-210, 1984.

[75] J. Gasser and H. Leutwyler, "Chiral perturbation theory: expansions in the mass of the strange quark," Nuclear Physics B, vol. 250, no. 1-4, pp. 465-516, 1985.

[76] J. Goldstone, "Field theories with "superconductor" solutions," Il Nuovo Cimento, vol. 19, no. 1, pp. 154-164, 1961. 
[77] J. Goldstone, A. Salam, and S. Weinberg, "Broken symmetries," Physical Review, vol. 127, no. 3, pp. 965-970, 1962.

[78] H. Leutwyler, "Principles of chiral perturbation theory," in Hadron Physics 94-Topics on the Structure and Interaction of Hadronic Systems, V. E. Herscovitz, C. A. Z. Vasconcellos, and E. Ferreira, Eds., p. 1, World Scientific Publishing, Singapore, 1995.

[79] R. V. Lange, "Goldstone theorem in nonrelativistic theories," Physical Review Letters, vol. 14, no. 1, pp. 3-6, 1965.

[80] R. V. Lange, "Nonrelativistic theorem analogous to the Goldstone theorem," Physical Review, vol. 146, no. 1, pp. 301-303, 1966.

[81] G. S. Guralnik, C. R. Hagen, and T. W. B. Kibble, "Broken symmetries and the Goldstone theorem," in Advances in Particle Physics, R. L. Cool and R. E. Marshak, Eds., vol. 2, p. 567, John Wiley \& Sons, New York, NY, USA, 1968.

[82] H. B. Nielsen and S. Chadha, "On how to count Goldstone bosons," Nuclear Physics B, vol. 105, no. 3, pp. 445-453, 1976.

[83] H. Leutwyler, "Nonrelativistic effective Lagrangians," Physical Review D, vol. 49, no. 6, pp. 3033-3043, 1994.

[84] H. Leutwyler, "On the foundations of chiral perturbation theory," Annals of Physics, vol. 235, no. 1, pp. 165-203, 1994.

[85] P. Hasenfratz and F. Niedermayer, "Finite size and temperature effects in the AF Heisenberg model," Zeitschrift für Physik B, vol. 92, no. 1, pp. 91-112, 1993.

[86] N. D. Mermin and H. Wagner, "Absence of ferromagnetism or antiferromagnetism in one- or two-dimensional isotropic Heisenberg models," Physical Review Letters, vol. 17, no. 22, pp. 1133-1136, 1966.

[87] P. Kopietz and S. Chakravarty, "Low-temperature behavior of the correlation length and the susceptibility of a quantum Heisenberg ferromagnet in two dimensions," Physical Review B, vol. 40, no. 7, pp. 4858-4870, 1989. 

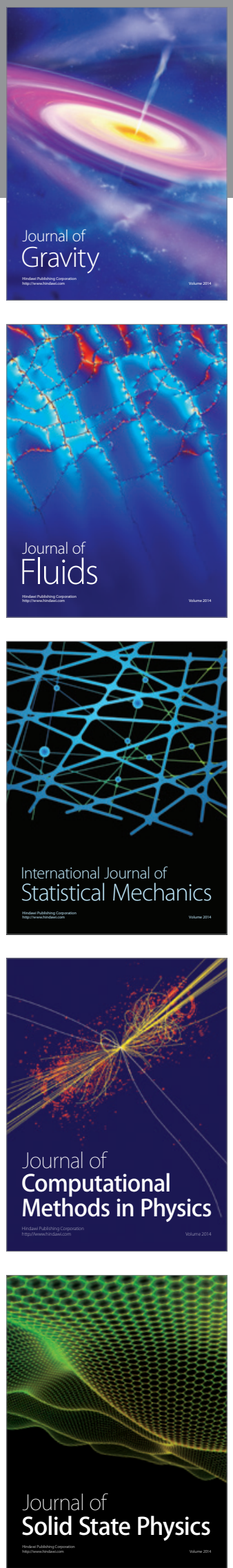

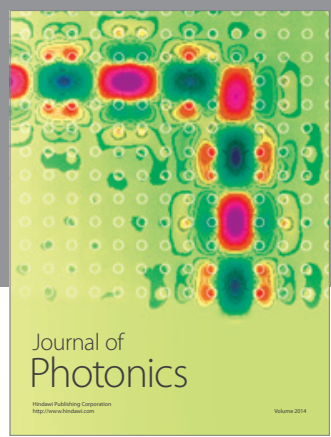

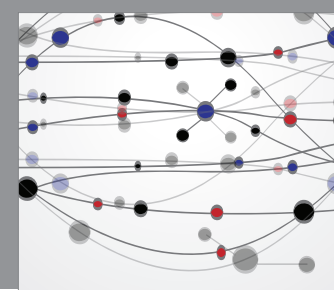

The Scientific World Journal

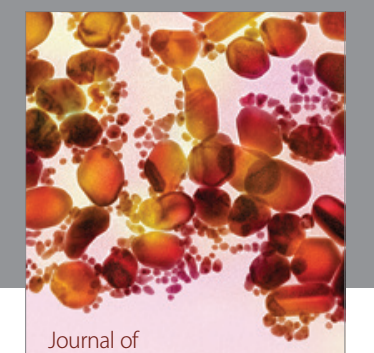

Soft Matter
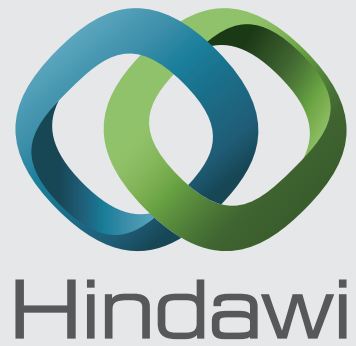

Submit your manuscripts at

http://www.hindawi.com
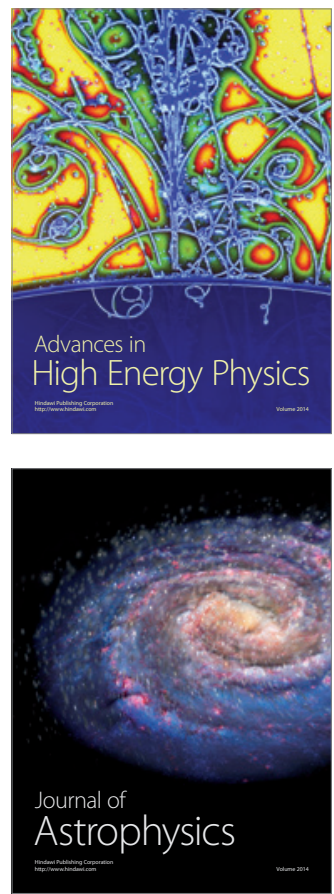
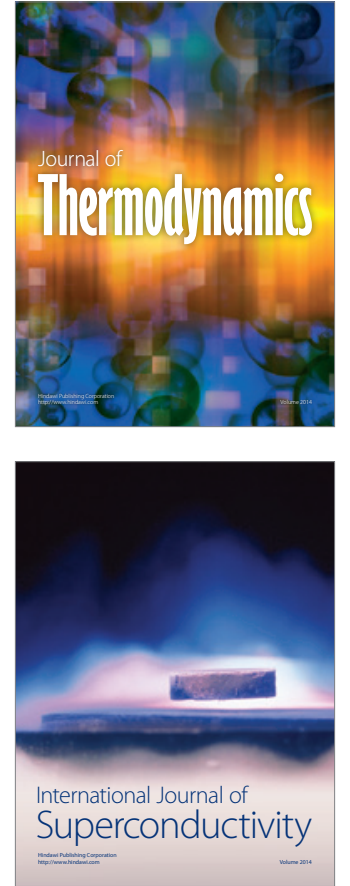
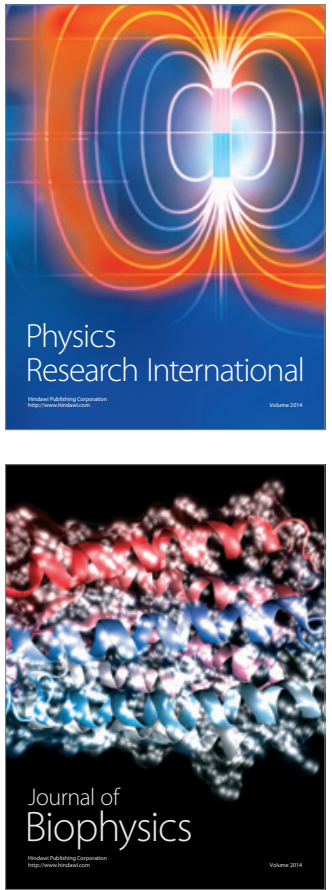
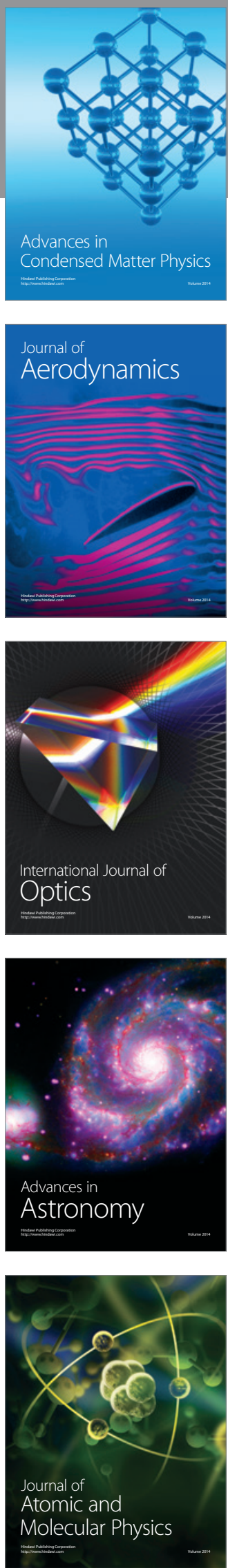